\title{
Resinas naturales de
}

\section{especies vegetales mexicanas: usos actuales y potenciales}

\author{
Natural resins of Mexican plant species: current and potential end-uses
}

\author{
Joaquín Antonio Quiroz Carranzal* y Miguel Alberto Magaña Alejandro²
}

\begin{abstract}
'Facultad de Ciencias Naturales, Universidad Autónoma de Querétaro Av. De las Ciencias S/N Juriquilla,

Querétaro, México. cenciart@yahoo.com.

* Autor de correspondencia. cenciart@yahoo.com.
\end{abstract}

\author{
² Universidad Juárez Autónoma de Tabasco. Km. 0.5, \\ carretera Villahermosa, Cárdenas entronque a Bos- \\ ques de Saloya. manglarujat@hotmail.com.
}

\section{RESUMEN}

Se revisa el estado actual de la información sobre la flora mexicana productora de resinas, así como sus aplicaciones actuales y potenciales. Los exudados vegetales están recuperando su lugar en el mercado internacional y México posee una amplia flora de coníferas, latifoliadas tropicales y de clima templado, árboles, arbustos y trepadoras, las cuales en mayor o menor cantidad producen resinas que tienen una amplia aplicación en actividades humanas. Los exudados vegetales son considerados productos forestales menores (PFM) o productos forestales no maderables (PFNM), y pudieran alcanzar o tal vez superar el valor económico de la producción maderera. Las 28 familias botánicas mencionadas en esta revisión están representadas ampliamente en la flora mexicana, en ellas están incorporados 79 géneros, los cuales incluyen especies que está documentado en la literatura botánica que producen resinas. Es necesario conocerlas profundamente para identificar nichos de oportunidad sea en la industria alimentaria, el curtido de pieles, la elaboración de barnices, la de preservadores o de aditivos de maderas, ya que pueden ser alternativas viables para el manejo sostenible de bosques y selvas, siempre y cuando se aprovechen para mejorar las condiciones de los productores y de aquellos trabajadores que participan en su transformación.

Palabras Clave: exudados vegetales, flora mexicana, industria forestal, manejo sostenible, productos forestales no maderables.

\section{ABSTRACT}

The current state of information on the resin producer Mexican flora, as well as its present and potential applications are reviewed. Plant exudates are regaining their place in the international market and Mexico has a rich coniferous flora, tropical hardwoods and temperate vines, trees, shrubs, and climbing plants which produce resins in varying amounts, having wide application in human activities. Plant exudates are considered minor forest produce (MFP) or non-timber forest products (NTFPs), and could reach or perhaps exceed the economic value of timber production. The 28 plant families mentioned in this review are widely represented in the Mexican flora; they belong to 79 genera which include species that produce resins and are documented in the technical literature. It is necessary to know them deeply in order to identify niche opportunities whether it is in the food industry, leather tanning, processing of varnish, wood preservatives or additives, as they can be viable for the sustainable management of forests and jungles, provided they are used to improve conditions for producers and workers who participate in its transformation.

KEYWORDS: plant exudates, Mexican flora, forest industry, sustainable management, non-timber forest products.

\section{INTRODUCCIÓN}

Los exudados de las maderas, parte de los denominados productos forestales menores (PFM) o productos forestales no maderables (PFNM), entre los cuales se incluyen gomas, resinas, mucílagos, aceites esenciales, aceites de semillas, alcaloides, extractos medicinales y tintes, se vienen usando desde la antigüedad en la alimentación humana, en los procesos mágico-religiosos, en terapias curativas, en teñido de fibras vegetales o animales y en preservación de madera, entre otros (Murugesan et al., 2011). 
Algunos de los exudados de mayor importancia por su valor económico o tradicional son las gomas como el chicle, el caucho y el látex; las resinas de coníferas para elaboración de trementina o de burseras como el copal, la mirra y el incienso; los aceites esenciales de lináloe, los taninos para la curtiduría, entre otros. México posee una amplia diversidad de especies arbóreas de coníferas y latifoliadas o de hoja ancha tropicales y de clima templado (Pennington y Sarukhán, 2005; Gernandt et al., 2014) que producen resinas y gomas, su extracción y aprovechamiento tienen una vasta perspectiva; algunos de los usos actuales son en la industria alimentaria, la farmacéutica, bebidas alcohólicas, tintes, pinturas y barnices. Potencialmente pudieran aplicarse en la conservación y restauración de bienes muebles de madera y textiles, o como aditivo para minimizar los cambios dimensionales de la madera generados por la humedad, sobre todo en instrumentos musicales de cuerda pulsada, frotada, de viento, entre otros.

En el caso de la madera, su deterioro se presenta por la influencia combinada de factores bióticos y abióticos como la humedad y la temperatura, especialmente el agua juega un papel importante en dicho proceso, ya que provoca cambios dimensionales como la contracción e hinchamiento de la madera y ofrece las condiciones para el crecimiento de microorganismos que, subsecuentemente, dañan la madera (Echenique-Manrique y Robles, 1993). Estos fenómenos ocurren debido a que la madera está compuesta principalmente de polímeros con grupos hidroxilo altamente hidrofílicos, los cuales facilitan la adhesión del agua por medio de puentes de hidrógeno. El uso de resinas naturales no hidro-solubles como preservadores de la madera deberá buscar que estas puedan impregnar y recubrir la pared celular para evitar que el vapor de agua o el agua líquida logren humedecerla y con ello minimizar los cambios dimensionales y el establecimiento de microorganismos (Hyvönen et al., 2005).

Hasta la fecha, los procesos de preservación de madera usan de forma convencional productos tóxicos para los seres humanos y dañinos al ambiente. Sin embargo, como señalan Brand et al. (2006), la polimeriza- ción de la pared celular de las maderas mediante el uso de resinas naturales pudiera dar protección interna a las paredes celulares con productos no tóxicos, insolubles en el agua. Esto reduciría la problemática con un bajo impacto ambiental (González-Laredo et al., 2015).

A los polímeros naturales, como las resinas, se les ha comenzado a llamar polímeros bioactivos, particularmente aquellos no hidro-solubles porque además de impedir la adsorción del agua, limitan el crecimiento de microorganismos. Si el uso de polímeros bioactivos se demostrara viable económicamente en la conservación de la madera se podrían resolver diversos problemas relacionados con el uso y durabilidad de la madera sin afectar el ambiente y la salud humana (Strategis, 2004, citado por Brand et al., 2006).

\section{Las resinas}

Las resinas se clasifican en sintéticas y naturales, las naturales en resinas típicas como el copal y la colofonia, sus componentes primarios son terpenoides y compuestos fenólicos; las gomo-resinas como la mirra y la asfétida, son polisacáridos; las óleo-resinas como copaiba y trementina contienen ácidos grasos y glicerol; los bálsamos como el estoraque y el bálsamo de Perú contienen fenoles y ésteres; y las gluco-resinas como la jalapa y el podófilo, tripertenos, entre otras sustancias (Romnah de la Vega, 1985) (Tabla 1). También se clasifican en resinas duras y suaves, las primeras como el copal, son solubles en alcohol y con un relativo alto punto de fusión, aunque también hay solubles en aceites y agua; por otra parte, las resinas suaves u óleo-resinas son aquellas que tienen un alto contenido de aceites (FAO, 1995).

La producción de resinas en México ocupa el tercer rubro de los denominados Productos Forestales No maderables (PFNM), con una producción que según Tapia y Reyes (2008), supera las 30000 toneladas anuales, pero las cifras de la Semarnat (2014) señalan cerca de 20000 toneladas anuales, representando $13 \%$ de los PFNM. Aproximadamente $90 \%$ de estas resinas corresponde a especies de Pinaceae, le sigue las de Burseraceae y Sapotaceae, pero no existen registros sobre producción de resinas de otras familias botánicas. 
TAвla 1. Características de algunas resinas.

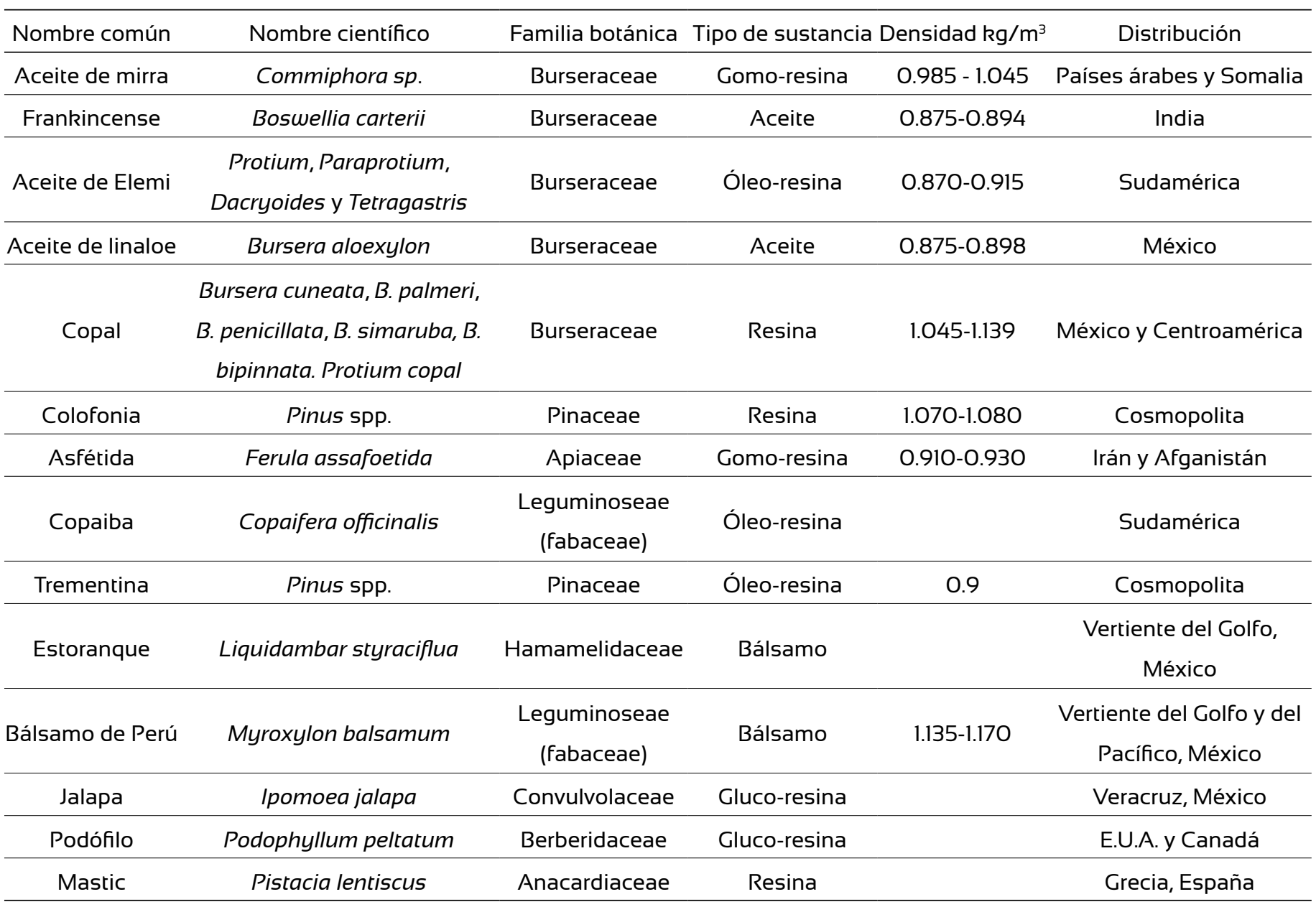

Fuente: Rossignon, 1869; Parry, 1921; Romnah de la Vega, 1985; Rzedowski y Guevara, 1992; Rüdiger et al., 2007

Para tener una comparación cuantitativa, se puede mencionar la producción de resinas en Etiopía, país que exporta 16000 toneladas anuales de gomas y resinas de Leguminoseae y Burseraceae (goma arábiga, frankincense, mirra y apopanax) con un valor de más de 20 millones de dólares; por otra parte la Comunidad Europea importa cerca de 30000 toneladas de este tipo de gomas y resinas (Tadesse et al., 2007).

\section{OBJETIVOS}

Con base en lo anterior se consideró necesario realizar una revisión bibliográfica sobre la diversidad vegetal de especies mexicanas productoras de resinas, su capa- cidad de producción, su uso actual y potencial, para aprovecharlas de forma sustentable.

\section{MATERIALES Y MÉTODOS}

Se realizó una revisión bibliográfica exhaustiva, utilizando artículos científicos, bases de datos de internet y libros físicos y electrónicos, como fuente documental para sistematizar la información sobre las familias botánicas, sus géneros $\mathrm{y}$ en algunos casos las especies que producen resinas, haciendo énfasis en la flora cuya distribución geográfica cubre desde el sur de los Estados Unidos de Norteamérica hasta Centro, Sudamérica y el Caribe continental, particularmente aquellos taxones que se distribuyen en México. 


\section{ResultadOS}

A continuación se mencionan las familias botánicas, géneros y en algunos casos las especies productoras de resinas, cuya distribución geográfica incluye México u otros países de la región, documentados en la literatura especializada.

\section{Anacardiaceae}

Reúne 65 a 75 géneros y entre 600 y 700 especies, está representada en México por 20 géneros y 62 especies, tanto nativas como introducidas, las especies de mayor importancia económica de esta familia son el mango (Mangifera indica L.), el ciruelo (Spondias purpurea L.), el pistacho (Pistacia vera L.) y el marañon (Anacardium occidentale L.). (Rzedowski y Calderon de Rzedowski, 1999). Los géneros que incluyen especies productoras de resinas, presentes en América y México se listan en la tabla 2.

TABLA 2. Géneros de Anacardiaceae productores de resinas

\begin{tabular}{cc}
\hline Género & Área de distribución \\
\hline Anacardium & Trópico americano incluido México \\
\hline Comocladia & Trópico americano incluido México \\
\hline Mangifera & Trópico americano incluido México \\
\hline Metopium & México y Sudamérica \\
\hline Pistacia & México y Guatemala \\
\hline Pseudosmodingium & México \\
\hline Schinus & Trópico americano incluido México \\
& Schinus molle, originario de Perú, \\
escapado del cultivo en México.
\end{tabular}

Fuente: Langeheim, (2003)

\section{Apocynaceae}

Incluye 215 géneros y 2100 especies de distribución pantropical, la mayoría de sus integrantes producen látex lechoso. En México está representada por 50 géneros y 385 especies. Entre los géneros más comunes están Thevetia y Gonolobus. Su mayor diversidad se ubica en las vertientes Atlántica y Pacífica: Campeche, Chiapas, Guerrero, Oaxaca y Veracruz (Juárez-Jaimes et al. 2007).

\section{Araceae}

Agrupa 105 géneros y aproximadamente 3300 especies, distribuidas en las regiones tropicales, subtropicales y templadas del hemisferio norte. En México se conocen 13 géneros y 109 especies nativas o naturalizadas, además cinco géneros y siete especies han sido introducidos (Croat y Carlsen, 2003). Los géneros presentes en América, productores de resinas, son: Monstera y Philodendron (Langeheim, 2003).

\section{Asteraceae}

Familia conformada con cerca de 950 - 1450 géneros y entre 20000 y 30000 especies en el mundo, en México se han documentado 373 géneros con 3080 especies nativas (Redonda-Martínez y Villaseñor-Ríos, 2011). Según Langeheim (2003), los géneros de esta familia, presentes en América, que producen resinas son: Grindelia, Gutierrezia, Haplopappus, Heterotheca, Brickellia, Tagetes, Balsamorhiza, Flourensia, Helianthus, Parthenium y Silphium.

\section{Berberidaceae}

Incluye 15 géneros y 650 especies (Mecham, 1980; Loconote, 1993), se distribuye en zonas templadas y andinas de Sudamérica. Particularmente de los rizomas y raíces de la especie Podophyllum peltatum se obtiene un extracto etanólico de lignanoglicosidos y flavonoides.

\section{Betulaceae}

Familia que incluye seis géneros y aproximadamente 100 especies. Se distribuye principalmente en la regiones templadas septentrionales, algunos géneros se encuentran en las zonas montañosas tropicales (Nee, 1981). Según Langeheim (2003), los géneros Alnus y Ostrya producen resinas. 


\section{Burseraceae}

Agrupa 17 géneros y 548 especies, distribuidas en América tropical, África, Ceilán, Madagascar, Arabia, la India y Malasia. En la región neotropical esta familia está representada por 228 especies agrupadas en ocho géneros (Rzedowski y Guevara, 1992; Rüdiger et al., 2007). Los géneros de Burseraceae presentes en México son: Protium (con una especie), Bursera (con 80 spp.), Commiphora (2 spp.), Beiselia (una especie) y Terebinthus (una especie), (Cházaro et al., 2010). El género Bursera reúne más de 100 especies distribuidas desde el sur de los Estados Unidos hasta Sudamérica, con una amplia diversidad en la vertiente pacífica de México.

Los copales más utilizados históricamente por las poblaciones indígenas de México se obtienen de Protium copal, en la vertiente del Golfo de México, desde San Luis Potosí hasta Quintana Roo y Chiapas. Mientras que las poblaciones indígenas de la vertiente del Pacífico: Morelos, Puebla, Oaxaca y Michoacán extraen la resina de Bursera bipinnata, B. glabrifolia y B. copallifera (Cruz et al., 2006; Cházaro, et al., 2010; López, 2012). El aceite "lináloe" se extrae de B. Aloexylon y B. linaloe (Rzedowski y Guevara, 1992; Cházaro, et al., 2010). Por otra parte en el neotrópico están presentes los géneros Dacryodes, Tetragastris y Trattinnickia, los cuales también producen resinas (Langeheim, 2003).

El copal es una resina generada por células resiníferas de diversas especies de Burseraceae, la cual ha sido utilizada desde la antigüedad por diversas culturas para la elaboración de barnices, colorantes, fijadores, impermeabili zantes de embarcaciones, repelentes de insectos, saumerios y en medicina tradicional para incrustaciones dentales, emoliente, antiséptico y analgésico (Rüdiger et al., 2007). Además de los usos antes mencionados, Rüdiger $e t$ al. (2007) señalan que el copal posee propiedades hidrófugas y de protección contra los procesos oxidativos, reconociéndose como un recurso natural aprovechable para incrementar la vida útil de diversos productos.

La resina copal está compuesta de monoterpenos, diterpenos y flavonoides, con una adhesividad entre 0.5 $\mathrm{MPa}$ a $6 \mathrm{MPa}$, posee propiedades bactericidas, antimicro- bianas, desinfectantes y antivirales (Orta, 2007). Los monoterpenos son los principales componentes de los aceites esenciales, los diterpenos son sustancias hidrófugas y poco volátiles, se denominan resinas ácidas, las cuales tienden a cristalizar por polimerización oxidativa; por su parte los flavonoides son sustancias vegetales que permiten a las plantas resistir la foto-oxidación de la luz ultravioleta (Higuchi, 1997). Según González (1996), en un estudio sobre uso de taninos como preservantes de madera, señala que los flavonoides de los taninos forman enlaces covalentes con el material celulósico de la madera mejorando algunas de sus propiedades físicas y otorgándole mayor estabilidad dimensional. Por su parte Nascimiento et al., (2013) señalan que los flavonoides ofrecen protección contra la radiación ultravioleta, patógenos y herbívoros, presentando un efecto importante sobre la durabilidad de la madera.

\section{Clusiaceae}

Familia con 27 géneros y 1090 especies con una distribución restringida a tierras bajas tropicales (Stevens, 2007). Según Langeheim, (2003), los géneros Clusia, Moronobea y Symphonia se distribuyen en el trópico americano y producen resinas.

\section{Convolvulaceae}

Está representada en México por 17 géneros y 217 especies (McDonald.1991). Ipomoea jalapa (L.) Pursh presente en México, produce una gluco-resina, la cual se ha usado en el tratamiento de diversos padecimientos.

\section{Dracaenaceae}

Está formada por dos géneros y 60 especies (González, 2006), se distribuye en las regiones subtropicales y tropicales, el único género nativo de América es Dracaena, cuyas especies se ha documentado producen resina (Langeheim, 2003).

\section{Euphorbiaceae}

Agrupa 320 géneros y 8700 especies. En México está representada por 43 géneros y 782 especies (Steinmann, 2002). 
El género Dalechampia, presente en zonas cálidas de América, está documentado como productor de resinas (Langeheim, 2003). De igual manera la especie Ricinus communis, es la planta de donde se extrae el aceite de Ricino.

\section{Hamamelidaceae}

Reúne 26 géneros y más de 100 especies distribuidas en América, Asia, África y Australia. En México existen cuatro géneros: Distylum, Hamamelis, Matudaea y Liquidambar (Carranza, 2004). Particularmente la especie Liquidambar styraciflua L. se conoce como quirámbaro, somerio, bálsamo, copalillo, copalme, estoranque, ícob, liquidámbar, nabá, ocotzotl, ocozote, suchete, techco y xochiocotzocquahuitl y está documentado que produce resina. Se distribuye desde el este de los Estados Unidos hasta Nicaragua. En México en la vertiente del Golfo, desde Tamaulipas, Veracruz, San Luis Potosí, Hidalgo, Puebla y por el Pacífico en Oaxaca y Chiapas (Pennington y Sarukhán, 2005). En el estado de Querétaro prospera en el noreste en los municipios de Jalpan de Serra, Landa de Matamoros, Pinal de Amoles y potencialmente se puede encontrar en San Joaquín. Su madera se usa para decorar interiores de habitaciones y su resina (copalme, estoranque) tienes usos en la medicina tradicional, en actividades ceremoniales y en la industria de la perfumería y cosmética. Las hojas contienen taninos y los árboles se cultivan en calles y jardines de ciudades con fines ornamentales (Carranza, 2004).

\section{Humiriaceae}

Comprende 8 géneros y 61 especies distribuidas en la zona neotropical, principalmente de Nicaragua al sur de Brasil (Petean, 1997). Langeheim (2003) menciona que el género Humiria produce resina.

\section{Hydrophyllaceae}

Reúne entre 18 y 20 géneros y unas 250 a 300 especies, de distribución casi cosmopolita pero preferentemente en las regiones templadas y tropicales del hemisferio norte. En México se han identificado cuatro géneros y 15 especies
(Pérez-Calix y Carranza, 2005). El género Eriodictyon, presente en el suroeste de Norteamérica, es considerado productor de resina (Langeheim, 2003).

\section{Leguminosae (Fabaceae)}

Es una de las familias botánicas más abundante del reino vegetal. Según Sousa et al. (2004), reúne 18 mil especies, las cuales están distribuidas en tres subfamilias: Mimosoideae, Cesalpiniodeae y Faboideae. En México se han registrado 1850 especies, de las cuales, 633 son arbustivas y arbóreas (Sousa et al., 2004). Los géneros que según Langeheim (2003) producen resinas son: Eperua, Hymenanea, Prioria y Myroxylon.

Particularmente la especie Myroxylon balsamum se distribuye desde México hasta Centroamérica y en México desde Veracruz y Oaxaca hasta el sur de la Península de Yucatán y en la vertiente del Pacífico desde Nayarit a hasta Chiapas. Produce una óleo-resina insoluble en agua y soluble en etanol (90\%), acetona, cloroformo y otros solventes; posee entre $45 \%$ y $64 \%$ de aceites volátiles y $20 \%$ a $25 \%$ de resina (Andre, 2003)., la cual tiene propiedades cicatrizantes, antiinflamatorias y analgésicas.

Otros géneros de Fabaceae representados en la flora mexicana, sobre los que se ha documentado que producen resinas son:

El género Acacia, que reúne 1200-1400 especies distribuidas en el mundo, de las cuales 160-200 se encuentran en América. En México, el género está representado por 73-84 especies, de las cuales 34 son endémicas (Rico, 2001). Muchas de ellas producen resinas. En la porción centro-norte del país se reconocen 15 especies: A. cornígera, A. pennatula, A. amentaceae, A. coulteri, A. farnesiana, A. constricta, A. schaffneri, A. anisophylla, A. sororia, A. reniformis, A. parviflora, A. fusicarpa, A. berlanderi y A. amabilis (Andrade et al., 2007) y el género Havardia reúne 8 especies desde el sur de Estados Unidos de América hasta Centroamérica, Colombia y las Antillas. Siete de estas especies están presentes en México.

El género Prosopis contiene 40 especies distribuidas en regiones áridas y semiáridas de Norte y Sudamérica, África y Asia. En México se encuentran 10 especies: $P$. 
palmeri, $P$. reptans var. cineroscens, $P$. pubescens, $P$. articulata, P. laevigata, P. tamaulipana, $P$. velutina, $P$. juliflora, P. glandulosa var. típica, P. glandulosa var. torreyana, y P. mexicanum (De La Torre-Almaráz et al, 2009), también los géneros Parkinsonia, Swarzia, Apoplanesia y Ateleia incluyen especies productoras de resinas (Flores, 2001).

\section{Moraceae}

Esta familia posee 37 géneros y 1100 especies de distribución mundial, principalmente tropical con algunos representantes en regiones templadas y subtropicales. Diversos géneros presentan abundantes células laticíferas (Rohwer y Berg, 1993). Particularmente Ficus se distribuye en zonas tropicales y produce resinas (Langeheim, 2003). De igual manera otra de las especies importante productora de resina es Castilla elastica, de la cual los mayas obtenían una resina para la elaboración de las pelotas utilizadas en los juegos rituales.

\section{Pinaceae}

En México esta familia está representada por 4 géneros, los cuales agrupan 61 especies: Picea (3), Pinus (44), Abies (13) y Pseudostuga (1) (Gernandt y Pérez de la Rosa, 2014). En el año 1991 se produjeron en México 30000 toneladas de resina cruda de pinos, 22000 de brea y 4 000 de aguarrás, principalmente en los estados de Michoacán, Jalisco, Estado de México, Durango, Nuevo león y Oaxaca (Arias y Chávez, 2006). Las especies de mayor producción de resina son: pino trompillo (P. oocarpa), pino chino (Pinus leiophylla), pino ocote (P. lawsonii), pino llanero ( $P$. herrerai), pino canis (P. tenuifolia), ocote (P. montezumae), pino real (P. ponderosa) y pino rojo ( $P$. pringlei) (Romanh de la Vega, 1985.; Arias y Chávez, 2006) (Tabla 3).

Los productos derivados de la extracción de resinas de pinos son: la trementina que se usa en la elaboración de lacas, preparación de lacres, ungüentos, emplastos, bálsamos y expectorantes; la brea o colofonia se utiliza en la

Tabla 3. Pinos productores de resina.

\begin{tabular}{|c|c|c|}
\hline Nombre común & Nombre científico & Distribución \\
\hline Pino trompillo & P. oocarpa & $\begin{array}{c}\text { México, Sonora, Chihuahua, Sinaloa, Zacatecas, Durango, Nayarit, Jalisco, Michoacán, } \\
\text { Guerrero, Oaxaca, Chiapas, Morelos, Puebla, Hidalgo, Tlaxcala y Veracruz. }\end{array}$ \\
\hline Pino chino & P. leiophylla & $\begin{array}{l}\text { Chihuahua, Durango, Zacatecas, Jalisco, Sinaloa, Nayarit, Colima, Michoacán, México, } \\
\text { Tlaxcala, Puebla, Morelos, Veracruz y Oaxaca. }\end{array}$ \\
\hline Pino ocote & Pinus lawsonii & Jalisco, Michoacán, México, Morelos, Puebla, Guerrero, Oaxaca, Hidalgo y Morelos. \\
\hline Pino llanero & $\begin{array}{l}\text { P. teocote o } \\
\text { P. herrerai }\end{array}$ & $\begin{array}{l}\text { Desde el sur de Chihuahua hasta Chiapas, desde Coahuila y Nuevo León hasta Hidalgo, } \\
\text { Estado de México y Puebla. }\end{array}$ \\
\hline Pino canis & $\begin{array}{l}\text { P. maximinoi o } \\
\text { P. tenuifolia }\end{array}$ & $\begin{array}{l}\text { Jalisco, Michoacán, Guerrero, Puebla, Tlaxcala, Veracruz, Hidalgo, Morelos, Oaxaca y } \\
\text { Chiapas. }\end{array}$ \\
\hline Pino, ocote & P. montezumae & $\begin{array}{c}\text { Coahuila, Nuevo León, Tamaulipas, Hidalgo, Tlaxcala, Puebla, Veracruz, México, Michoa- } \\
\text { cán, Jalisco, Guerrero, Oaxaca, Chiapas, Colima, Morelos, Querétaro, Zacatecas y Distrito } \\
\text { Federal. }\end{array}$ \\
\hline $\begin{array}{l}\text { Pino ponderosa, } \\
\text { pino real }\end{array}$ & $\begin{array}{l}\text { P. ponderosa o } \\
\text { P. Engelmannii }\end{array}$ & Chihuahua, Sonora, Sinaloa, Durango y Zacatecas. \\
\hline Pino rojo & P. pringlei & Michoacán, Guerrero, México, Morelos, Puebla y Oaxaca. \\
\hline
\end{tabular}

Fuente: Romanh de la Vega (1985); Narave y Taylor (1997); Arias y Chávez (2006); Conafor (2014). 
elaboración de pinturas, barnices, lacas, lubricantes, aceites y betún de zapatos; el aguarrás o esencia de trementina como solvente de pinturas y barnices, secador de esmaltes, sellador de madera, preparación de lacas, fabricación de insecticidas, desinfectantes, colorantes, jabones, medicinas y perfumes (Romanh de la Vega, 1985).

\section{Piperaceae}

Es una familia tropical con cinco géneros y 2000 - 3000 especies (Tebbs, 1993). 104 especies están presentes en Centro y Sudamérica y, particularmente en México, la familia está representada por 13 especies (Quijano-Abril et al., 2006) y el género Piper se menciona como productor de resina (Langeheim, 2003).

\section{Podocarpaceae}

Incluye entre 7 y 17 géneros con aproximadamente 170 especies distribuidas principalmente en las regiones tropicales y subtropicales de ambos hemisferios. En México solo está representada por el género Podocarpus (Zamudio, 2002). En Centro y Sudamérica los géneros productores de resinas son: Prummopitys, Retrophyllum y Saxegothaea (Langeheim, 2003).

\section{Rosaceae}

Esta familia reúne de 90 a 100 géneros y 2000 a 3000 especies, representadas en todo el mundo, principalmente en regiones templadas y subtropicales del hemisferio norte. Incluye especies de gran importancia económica como Prunus armeniaca L. (chabacano), P. domestica L. (ciruelo), Pyrus communis L. (peral), P. malus L. (manzano), entre otros (Rzedowski y Calderón de Rzedowski, 2005). Según Langeheim (2003), especies del género Prunus producen resinas.

\section{Rubiaceae}

Agrupa 650 géneros y 13000 especies. En México está representada por 103 géneros y 585 especies, con distribución en las regiones tropicales y subtropicales del país (Diego, 2007). Esta familia incluye especies de gran importancia económica como el café Coffea arabica, la quina Cinchona spp. y la ipecacuana Cepahelis ipecacuana (Brot.) L. Anderson. México posee 15 géneros y 278 especies endémicas. Los estados con mayor número de especies endémicas de esta familia son: Oaxaca, Guerrero, Veracruz y Chiapas (Diego, 2007). Según Langeheim (2003), los géneros Carphalea y Coutarea, presentes en el trópico americano, poseen especies productoras de resinas.

\section{Rutaceae}

La familia se compone de 155 géneros y 1500 especies de distribución mundial, pero más abundante en los trópicos y subtrópicos de América, África y Australia. Las especies de gran importancia económica de esta familia son del género Citrus (naranja, limón, mandarina). Una característica de la familia es la presencia de aceites en tallos, hojas y frutos (Zamora, 2006). Según Langeheim (2003), los géneros Amyris y Xanthoxylon, presentes en el trópico americano, producen resinas.

\section{Salicaceae}

Esta familia incluye dos géneros y más de 300 especies. En México hay representantes de los dos géneros Populus y Salix (Carranza, 1995), los cuales son considerados productores de resina (Langeheim, 2003).

\section{Sapotaceae}

La familia está constituida por 53-54 géneros y más de 1110-1175 especies. En México está representada por 5 géneros y 38 especies. Se distribuye en el sur-sureste de México, desde Veracruz hasta Yucatán y Quintana Roo; en el Bajío y regiones adyacentes, así como en Puebla y Oaxaca. La especie más conocida por su fruta comestible es el chicozapote, Manilkara zapota, de donde se obtiene una resina que es utilizada para la elaboración del chicle (Carranza, 2005; Newman, 2008).

\section{Scrophulariaceae}

Está conformada por 65 géneros y cerca de 1700 especies. Aunque su distribución es cosmopolita, es más diversa en las regiones templadas y en las montañas 
intertropicales (Pérez-Calix, 2011). El género que está documentado como productor de resina es Mimulus (Langeheim, 2003).

\section{Styracaceae}

Familia con alrededor de 12 géneros y cerca de 200 especies, distribuidas en regiones tropicales y templadas de ambos hemisferios. El género Styrax reúne entre 160 y 190 especies, entre las que destacan S. officinalis L., S. benzoin Dryand. y $S$. tonkinensis Craib ex Hartwich, de las cuales se obtienen bálsamos para inciensos. En México se reconocen cinco especies de este género (Carranza, 1993).

\section{Taxodiaceae}

Esta familia incluye 10 géneros con 16 especies, distribuidas principalmente en las regiones templadas del hemisferio norte (Carranza, 1992). El género Taxodium es considerado productor de resina (Langeheim, 2003). La especie Taxodium mucronatum está presente en México y se conoce como sabino o ahuehuete.

\section{Zygophyllaceae}

Está constituida por 27-30 géneros y alrededor de 250 especies en el mundo. En México está representada por seis géneros y 18 especies (Medina-Lemos, 2012). Los géneros presentes en América y México productores de resinas se listan en la tabla 4 .

TABla 4 . Géneros de Zygophyllaceae productores de resinas

\begin{tabular}{cc}
\hline Guaiacum & América templada cálida \\
Larrea & Suroeste de América del Norte y Sudamérica \\
Metharme & Chile \\
Pintoa & Chile \\
Plectocarpa & Sudamérica templada \\
Porlieria & México, Andes \\
Sericoides & Norte de México \\
\hline
\end{tabular}

Fuente: Langeheim, (2003)

\section{DISCUSIÓN}

En esta revisión se identificaron 28 familias botánicas que incluyen 79 géneros, donde está agrupada al menos una especie productora de resinas y cuya distribución geográfica incluye México, países Centroamericanos, Sudamericanos y el Caribe continental (Tabla 5). Solamente existe información disponible sobre las características químicas, físicas y biológicas de las resinas de especies de algunos géneros como Pistacia, Schinus, Podophyllum, Protium, Bursera, Convolvulus, Liquidambar, Hymenanea, Acacia, Myroxylon, Havardia, Prosopis, Pinus, Manilkara y Styrax. Los usos principales son medicinales, mágico-religiosos, aditivos de alimentos, golosinas, barnices y solventes. Pero es escasa o nula la información documental sobre el uso de las resinas en aplicaciones no convencionales como la reducción de los cambios dimensionales y preservación de la madera.

\section{CONCLUSIONES}

La producción de resinas vegetales en México en el periodo 2004-2013 se mantuvo alrededor de las 20000 toneladas anuales, excepto en 2012 cuando superó las 65 000 toneladas. En 2013 se produjeron 18565 toneladas de resinas, $86 \%$ de estas las generó Michoacán, $11.07 \%$ el Estado de México, $1.26 \%$ Jalisco y el resto entre Campeche, Chiapas, Oaxaca y Quintana Roo (Semarnat, 2014).

México posee una flora muy diversa y la mayoría de las 28 familias botánicas mencionadas en esta revisión están representadas ampliamente, estas incluyen 79 géneros, los cuales está documentado incluyen especies productoras de resinas

El universo de especies productoras de resinas es muy basto, es necesario desarrollar estudios para conocer las características físicas y propiedades químicas de las resinas, sus posibilidades de producción y aplicación en la industria alimentaria, farmacéutica, en la elaboración de selladores y barnices, así como en la conservación de maderas, entre otras aplicaciones.

Particularmente el estudio y aplicación de resinas naturales en la conservación de bienes que forman parte del patrimonio cultural, pueden contribuir a desarrollar 
TAвla 5. Resumen de las familias y géneros con especies productoras de resinas presentes en México y áreas circunvecinas

\begin{tabular}{|c|c|c|c|c|c|}
\hline Familia & Género & Familia & Género & Familia & Género \\
\hline \multirow{9}{*}{ Anacardiaceae } & Anacardium & \multirow{5}{*}{ Burseraceae } & Protium & \multirow{2}{*}{ Moraceae } & Ficus \\
\hline & Comocladia & & Bursera & & Castilla \\
\hline & Mangifera & & Dacryoides & Pinaceae & Pinus \\
\hline & Metopium & & Tetragastris & Piperaceae & Piper \\
\hline & Pistacia & & Trattinnickia & \multirow{3}{*}{ Podocarpaceae } & Prummopitys \\
\hline & Pseudosmodingium & \multirow{3}{*}{ Clusiaceae } & Clusia & & Retrophyllum \\
\hline & Schinus & & Monorobea & & Saxegothaea \\
\hline & Tapirira & & Symphonia & Rosaceae & Prunus \\
\hline & Toxicodendron & Convolvulaceae & Convolvulus & \multirow{2}{*}{ Rubiaceae } & Carphalea \\
\hline \multirow{2}{*}{ Apocynaceae } & Thevetia & Taxodiaceae & Taxodium & & Coutarea \\
\hline & Gonolobus & Dracaenaceae & Dracaena & \multirow{2}{*}{ Rutaceae } & Amyris \\
\hline \multirow{2}{*}{ Araceae } & Monstera & Funhorbizrom & Dalechampia & & Xanthoxylon \\
\hline & Philodendron & cupnomidcede & Ricinus & \multirow{2}{*}{ Salicaceae } & Populus \\
\hline \multirow{11}{*}{ Asteraceae } & Grindelia & Hammaelidaceae & Liquidambar & & Salix \\
\hline & Gutierrezia & Humiricaeae & Humiria & Sapotaceae & Manilkara \\
\hline & Haplopappus & Hydrophyllaceae & Eriodictyon & Scrophulariaceae & Mimulus \\
\hline & Heterotheca & \multirow{11}{*}{$\begin{array}{c}\text { Leguminosae } \\
\text { (Fabaceae) }\end{array}$} & Eperua & Styracaceae & Styrax \\
\hline & Brickellia & & Hymenanea & \multirow{7}{*}{ Zygophyllaceae } & Guaicacum \\
\hline & Tagetes & & Prioria & & Larrea \\
\hline & Balsamorhiza & & Acacia & & Metharme \\
\hline & Flourensia & & Myroxylon & & Pintoa \\
\hline & Helianthus & & Havardia & & Plectocarpa \\
\hline & Parthenium & & Prosopis & & Porlieria \\
\hline & Silphium & & Parkinsonia & & Sericoides \\
\hline Berberidaceae & Podophyllum & & Swartzia & & \\
\hline \multirow{2}{*}{ Betulaceae } & Alnus & & Aplopanesia & & \\
\hline & Ostrya & & Ateleia & & \\
\hline
\end{tabular}

procedimientos que resuelvan problemáticas puntuales sin afectar la salud de aquellos que los manipulan y se sirven de ellos, ni producir residuos tóxicos que dañen los ecosistemas. Es necesario implementar estudios con la participación de etnobotánicos, fisiólogos vegetales, ecólogos, usuarios tradicionales y otros especialistas para dilucidar y entender los procesos, componentes, aprovechamientos de resinas vegetales y sobre todo la conservación del patrimonio biológico, para evitar su destrucción.
Es importante conocer lo más detalladamente posible las características y funciones ecológicas de las especies resiníferas, así como los procedimientos idóneos para su extracción, procesamiento y comercialización, para no repetir experiencias como la del chicle en el sur-sureste de México, la cual no contribuyó al desarrollo local y sí provocó el deterioro de los recursos naturales (Bancomext, 1981). Igual ha ocurrido en países como Etiopía, donde la explotación intensiva de resinas, el manejo inadecuado y otros procesos sinérgicos provocan el deterioro de los 
recursos naturales y no favorecen el desarrollo regional y local (Tadesse et al., 2007).

\section{REFERENCIAS}

Andrade M., G., G. Calderón de Rzedowski, S.L. CamargoRicalde, R. Grether, H.M. Hernández, A. Martínez-Bernal, L. Rico, J. Rzedowski y M. Sousa S. 2007. Subfamilia Mimosoideae. Flora del Bajío y de Regiones Adyacentes 150:1-229.

Andre R., J.R. 2003. Evaluación de procesos de purificación de resina del Bálsamo de Perú Myroxylon balsamum por medio de métodos físicos. Tesis para obtener el título de Ingeniero en Agroindustria. Escuela Agrícola Panamericana. Honduras. 45 p.

Arias T., A.A. y A. Chávez L. 2006. Resina: entre la madera y el desarrollo comunitario integral. Biodiversitas 65:1-7.

Bancomext. 1981. La crisis del chicle. Comercio Exterior 31(9):963-968.

Brand, M. A.; J. Aznaldo y J.C. Moreschi. 2006. Novos productos para o tratamiento preservante da madeira. Perspectivas da pesquisa e utilizacao. Floresta 36:129-138.

Carranza G., E. 1992. Taxodiaceae. Flora del Bajío y de Regiones Adyacentes 4:1-7.

Carranza G., E. 1993. Styracaceae. Flora del Bajío y de Regiones Adyacentes 21:1-13.

Carranza G., E. 1995. Salicaceae. Flora del Bajío y de Regiones Adyacentes 37:1-21.

Carranza G., E. 2004. Hamamelidaceae. Flora del Bajío y de Regiones Adyacentes 125:1-12.

Carranza G., E. 2005. Sapotaceae. Flora del Bajío y de Regiones Adyacentes 132:1-28.

Comisión Nacional Forestal. 2014. http://www.conafor.gob. $\mathrm{mx} /$ portal/index.php/temas-forestales/reforestacion/ fichas-tecnicashttp://www.conafor.gob.mx/portal/index. php/temas-forestales/reforestacion/fichas-tecnicas

Croat, T.B. y M. Carlsen. 2003. Araceae. Flora del Bajío y de Regiones Adyacentes 114:1-40

Cruz L., A.; L. Salazar M. y M. Campos O., 2006. Antecedentes y actualidad del aprovechamiento de copal en la Sierra de Huautla, Morelos. Revista de Geografía Agrícola 37:97-115.
Cházaro B., M., B.L. Mostul y F. García L. 2010. Los copales mexicanos (Bursera spp.). Bouteloua 7:57-70.

De La Torre-Almaráz, R., F.M. Cota-Trujillo, J.L. GarcíaRojas, J.E. Campos, y F. San-Martín. 2009. Etiología de la muerte descendente del mezquite (Prosopis laevigata L.) en la Reserva de la Biósfera del Valle de Zapotitlan, México. Agrociencia 43(2):197-208.

Diego P., N. 2007. Reseña de "Rubiaceae de México" de Borhidi A. Boletín de la Sociedad Botanica de México 81:147-148.

Echenique-Manrique, R. y F. Robles F. 1993. Ciencia y tecnología de la madera I. Universidad Veracruzana. p:9-17.

Flores, J.S. 2001. Leguminosae. Florística, etnobotánica y ecología. Etnoflora yucatanense. 320p.

FAO (Organización de las Naciones Unidas para la Alimentación y la Agricultura). 1995. Gums, resins and latexes of plant origin. Non-wood forest products No. 6. 20 p.

Gernandt, D.S. y J.A. Pérez de la Rosa. 2014. Biodiversidad de Pinophyta (coníferas) en México. Revista Mexicana de Biodiversidad 85:126-133.

González Laredo., R.F. 1996. Preservación de madera con taninos. Madera y Bosques 2(2):67-73.

González-Laredo, R.F., M. Rosales-Castro, N.E. Rocha-Guzmán, J.A. Gallegos-Infante, M.R. Moreno-Jiménez and J.J. Karchesy. 2015. Wood preservation using natural products. Madera y Bosques 21(Núm. esp.):63-76.

González, J. 2006. Dracaenaceae. Flora Digital de La Selva. Organización de Estudios Tropicales. http://sura.ots.ac. cr/local/florula2/families/DRACAENACEAE.pdf.

Higuchi, T. 1997. Biochemistry and molecular biology of Wood. Springer series in Wood science. $362 \mathrm{p}$.

Hyvönen, A.; P. Piltonen y J. Niinimäki. 2005. Biodegradable substance in wood protection. In: Anneli Jalkanen y Pekka Nygren, eds. 2005. Sustainable use of renewable natural resources - from principles to practices. University of Helsinki Department of Forest Ecology Publications 34. http://www.honeybee.helsinki.fi/mmtdk/ mmeko/sunare

Juárez-Jaimes, V., L.O. Alvarado Cárdenas y J.L. Villaseñor. 2007. La familia Apocynaceae sensu lato en México: diversidad y distribución. Revista Mexicana de Biodiversidad 78:459-482. 
Langenheim, J.H. 2003. Plant resins: chemistry, evolution, ecology and ethnobotany. Timber Press. Cambridge. 581 p

Loconote, H. 1993. Berberidaceae. In: K. Kubitzki, J.G. Rohwer, y V. Brittich, eds. The families and genera of vascular plants Vol. 2. Springer-Verlag. Berlin Heidelberg. p:147152.

López L., L. 2012. Humo aromático para los dioses: una ofrenda de sahumadores al pie del templo Mayor de Tenochtitlan. Instituto Nacional de Antropología e Historia. $142 \mathrm{p}$.

McDonald, A. 1991. Origin and diversity of mexican convolvulaceae. Anales Instituto de Biología. Serie Botánica 62(1):65-82

Mecham, C. 1980. Phylogeny of the Berberidaceae with an evaluation of classifications. Systematic Botany. American Society of Plants Taxonomist 5(2):149-172.

Medina-Lemos, R. 2012. Zygophyllaceae. Flora del Valle de Tehuacán-Cuicatlán108:1-18.

Murugesan, K., G., Ravi Kumar, S.H., Jain, S. Mohan, y S G. Nair. 2011. Wood exudates overview on aromatic gums and resins. Journal Indian Academic Wood Science $8(2): 72-75$

Nascimento, M.S., A.L.B.D. Santana, C.A. Maranhão, L.S. Oliveira y L. Bieber. 2013. Phenolic extractives and natural resistence of Wood. Intech. p:349-370.

Narave, F.H. y K. Taylor. 1997. Pinaceae. Flora de Veracruz 98:1-26.

Nee, M. 1981. Betulaceae. Flora de Veracruz 20:1-20

Newman, M.F. 2008. Sapotaceae Juss. Flora del Valle de Tehuacán-Cuicatlán 57:1-20

Orta A., M.N. 2007. Copal: microestructura, composición y algunas propiedades relevantes. Tesis para obtener el título de Ingeniero Químico Industrial. Escuela Superior de Ingeniería Química e Industrias Extractivas. Instituto Politécnico Nacional. México. 69 p.

Parry, E.J. 1921. The chemistry of essential oils and artificial perfumes. Scott, Greenwood and Son. Nueva York. 549 p.

Pennington, T.D. y J. Sarukhán. 2005. Árboles tropicales de México. Manual de identificación de las principales especies. Universidad Nacional Autónoma de México. Fondo de Cultura Económica. México. 521 p.
Pérez-Calix, E. 2011. Scrophulariaceae. Flora del Bajío y de Regiones Adyacentes 173: 1-25.

Pérez-Calix, E. y Carranza G., E. 2005. Hydrophyllaceae. Flora del Bajío y de Regiones Adyacentes 139: 1-55.

Petean B., C. 1997. Phylogenetic analysis of Humiriaceae with notes on the monophyly of Ixionanthaceae. Journal of Computational Biology 2(1):19-24

Quijano-Abril, M.A., R. Callejas-Posada y D.R. MirandaEsquivel. 2006. Areas of endemism and distribution patterns for neotropical piper species (Piperacea). Journal of Biogeography 33:1266-1278.

Redonda-Martínez, R. y J.L. Villaseñor-Ríos. 2011. Asteraceae Bercht. \& J. Presl. Flora del Valle de Tehuacán-Cuicatlán89:1-64.

Rico A., M.L. 2001. El género Acacia (Leguminosae, Mimosoideae) en el estado de Oaxaca, México. Anales del Jardín Botánico de Madrid 58(2):251-302.

Rohwe, J.G. y C.C. Berg. 1993. Moraceae. In: K. Kubitzki, J.G. Rohwer y V. Brittich, eds. The families and genera of vascular plants Vol. 2. Springer-Verlag Berlin Heidelberg. $\mathrm{p}: 438-453$.

Romanh de la Vega, C.F. 1985. Principales productos forestales no maderables de México. Universidad Autónoma de Chapingo. Estado de México. 561 p.

Rossignon, J. 1869. Manual de barnices y preparación de charoles, según los procederes más recientes. Librería de Rosa y Bouret. París. 226 p.

Rüdiger, A.L., A.C. Siani y V.F. Veiga Junior. 2007. The chemistry and pharmacology of the South American genus Protium Burm f. (Burseraceae). Pharmacognosy reviews $1(1): 93-104$

Rzedowski, J. y F. Guevara-Féfer, 1992. Burseraceae. Flora del Bajío y de Regiones Adyacentes 3:1-46.

Rzedowski, J. y G. Calderon de Rzedowski. 1999. Anacardiaceae. Flora del Bajío y de Regiones Adyacentes 78:1-52.

Rzedowski, J. y G. Calderon de Rzedowski. 2005. Rosaceae. Flora del Bajío y Regiones Adyacentes 135:1-157.

Semarnat (Secretaría de Medio Ambiente y Recursos Naturales). 2014. Anuario estadístico de la producción forestal 2013. Dirección General de Gestión Forestal y de Suelos. México. 221 p. 
Sousa S., M., R. Medina L., G. Andrade M., y M.L. Rico A. 2004. Leguminosas. Biodiversidad de Oaxaca. Instituto de Biología, Universidad Nacional Autónoma de México, Fondo Oaxaqueño para la Conservación de la Naturaleza, World Wildlife Fund. 346 p.

Steinmann, V. 2002. Diversidad y endemismo de la familia Euphorbiaceae en México. Acta Botánica Mexicana 61:61-93.

Stevens, P.F. 2007. Clusiaceae-Guttiferae. In: K. Kubitzki, ed. The families and genera of vascular plants Vol. 9. Springer-Verlag Berlin Heidelberg. p:48-66.

Tadesse, W., G. Desalegn y R. Alia. 2007. Natural gum and resin bearing species of Ethiopia and their potential applications. Investigación Agraria; Sistemas y Recursos Forestales 16(3):211-221.

Tapia-Tapia, E.C. y R. Reyes-Chilpa. 2008. Productos forestales no maderables en México: aspectos económicos para el desarrollo sustentable. Madera y Bosques 14(3):95-112.
Tebbs, M.C. 1993. Piperaceae. In: K. Kubitzki, J.G. Rohwer y V. Brittich, eds. The families and genera of vascular plants Vol. 2. Springer-Verlag. Berlin Heidelberg. p:516-520.

Zamora, N. 2006. Rutaceae. Flora digital de La Selva. Organización para Estudios Tropicales. Pp.1-4. http://sura.ots. ac.cr/local/florula2/families/RUTACEAE.pdf

Zamudio, S. 2002. Podocarpaceae. Flora del Bajío y Regiones Adyacentes 105:1-11.

Manuscrito recibido el 18 de agosto de 2014. Aceptado el 18 de mayo de 2015.

Este manuscrito se debe citar como:

Quiroz C., J.A.y M.A. Magaña A. 2015. Resinas naturales de especies vegetales mexicanas: usos actuales y potenciales. Madera y Bosques 21(3):171-183. 